\title{
CHARACTERIZATION OF CERTAIN GROUPOIDS AND LOOPS
}

\author{
PL. KANNAPPAN
}

Abstract. Groupoids (loops) possessing the left inverse property (lip) (also the right inverse property) and crossed-inverse (CI) loops are characterized as subvarieties of the variety of groupoids satisfying a single identity.

1. It is known that groups, Abelian groups, loops with the inverse property and the weak inverse property loops, can be defined by means of a single identity as a subvariety of the variety of groupoids [3], [5], [6], [4]. In this paper, we give characterizations for (i) groupoids (loops) with identity satisfying the left inverse property (lip) (also the right inverse property), (ii) crossed-inverse (CI) loops, as subvarieties of groupoids satisfying certain conditions.

A groupoid $G(\cdot)$ is said to have the left inverse property (lip), if, for every $x \in G$, there is at least one $a \in G$ such that $a \cdot(x y)=y$ for all $y \in G$. Consequently, for given $c, d \in G$, there is a unique $x \in G$ such that $c x=d$ holds. Similarly, $G(\cdot)$ possesses the right inverse property (rip) if, for each $x \in G$, there is at least one $b \in G$ such that $(y x) \cdot b=y$ for every $y \in G$. If $G(\cdot)$ has both lip and rip, then $G$ is said to have the inverse property and in this case $G(\cdot)$ is a quasigroup (see [2, p. 111]).

A groupoid $G(\cdot)$ in which the equation $y a=b$ has a unique solution for $y$, for every given pair $a, b \in G$ is called a right quasigroup. Similarly, if, for every pair $a, b \in G$, there is a unique $x$ such that $a x=b$ holds in $G(\cdot)$, then the groupoid is called a left quasigroup.

Loops $G(\cdot)$ in which the equivalent identities $(x y) \cdot \rho(x)=y$ or $\lambda(x) \cdot$ $(y x)=y$ hold for all $x, y \in G$ are called crossed-inverse (CI) loops, where $\rho(\lambda)$ is the right (left) inverse operator (see [1], [2]).

2. Let $G(\cdot)$ be a groupoid. We say that $G(\cdot)$ is an iso-lip (rip) groupoid (or loop) provided there is a lip (rip) groupoid (or loop) $G(*)$ with unity which is a principal isotope of $G(\cdot)$ such that $\cdot$ and * are connected by the relation $x * y=x \cdot \lambda(y)(x * y=\rho(x) \cdot y)$ for all $x, y \in G$, where $\lambda(\rho)$ is the left (right) inverse operator of $G(*)$.

Received by the editors July $26,1972$.

AMS (MOS) subject classifications (1970). Primary 20N05.

Key words and phrases. Groupoid, right (left) quasigroup, crossed-inverse loop, the (left or right) inverse property groupoid, isotope.

(c) American Mathematical Society 1973 
THEOREM 1. A necessary and sufficient condition that a groupoid $G(\cdot)$ is an iso-lip groupoid is that the following identity,

$$
(v v) \cdot[\{(t t) x \cdot(u u)(x y)\} \cdot(w w)]=y,
$$

or,

$$
[(v v) \cdot\{(t t) x \cdot(u u)(x y)\}] \cdot\left(w^{\prime} w\right)=y,
$$

holds for all $x, y, t, u, v, w \in G$.

Proof. We will consider only (1). The proof for $\left(1^{\prime}\right)$ is exactly the same as that of (1). First, we will show that $(\cdot)$ is left cancellative. Let $a \cdot s=a \cdot r$. Setting in (1), first $x=a, y=s$ and then $x=a, y=r$ and using $a \cdot s=a \cdot r$, we get $s=r$. Thus $(\cdot)$ is left cancellative.

Keeping $t, u, v, x$ and $y$ fixed in (1) and using the left cancellativity of $(\cdot)$, we obtain,

$$
w \cdot w=\text { constant }=e(\text { say }), \text { for all } w \in G .
$$

Now (1) becomes

$$
e \cdot[\{(e x) \cdot(e \cdot(x y))\} \cdot e]=y, \text { for all } x, y \in G .
$$

In (3), $y=e$ gives, by repeated use of (2) and the left cancellativity of $(\cdot)$,

$$
x e=x, \text { for all } x \in G .
$$

Now (3) can be rewritten as

$$
e \cdot[(e x) \cdot(e \cdot(x y))]=y, \text { for all } x, y \in G .
$$

Put $y=x$ in (5) to get, using (4), that

$$
e \cdot(e x)=x, \text { for all } x \in G .
$$

Define the mapping $\lambda: G \rightarrow G$, such that

$$
\lambda(x)=e \cdot x, \text { for all } x \in G .
$$

Then by $(6)$ and the left cancellativity of $(\cdot)$, it follows that $\lambda$ is a permutation of $G$.

Now define $G(*)$ as follows:

$$
x * y=x \cdot \lambda(y), \text { for all } x, y \in G .
$$

From (8), it is true that $G(*)$ is a principal isotope of $G(\cdot)$ and that $G(*)$ is a groupoid.

Further, using (6), (7) and (8), (5) becomes $\lambda[\lambda(x) \cdot \lambda(x y)]=y$, that is, 
$\lambda(x) *[x * \lambda(y)]=\lambda(y)$, that is,

$$
\lambda(x) *(x * y)=y, \text { for all } x, y \in G,
$$

which shows that $G(*)$ satisfies the lip.

Also, (8), (6) and (4) imply

$$
x * e=x=e * x, \text { for all } x \in G .
$$

Hence $G(\cdot)$ is an iso-lip groupoid.

Conversely, let $G(\cdot)$ be an iso-lip groupoid and let $G(*)$ be the corresponding lip groupoid with unity $e$ such that $(*)$ and $(\cdot)$ are connected by

$$
x * y=x \cdot \lambda(y) \text {, for all } x, y \in G,
$$

where $\lambda$ is the left inverse operator of $G(*)$. It is easy to see that $\lambda(x)$ is unique and $x * \lambda(x)=e=\lambda(x) * x$, for every $x \in G$. Thus, $\lambda^{2}(x)=x$, for every $x \in G$, showing thereby that $\lambda$ is a permutation of $G$. From (8), we get

$$
x \cdot y=x * \lambda(y) \text {. }
$$

So, $x \cdot x=x * \lambda(x)=e$, for all $x \in G, e \cdot y=\lambda(y)$ and $y \cdot e=y * e=y$, for all $y \in G$. Hence (2), (4) and (7) hold true. Use (8), (11), (2), (4) and (7) in (9) to get (1). This completes the proof of this theorem.

COROllary 1. Let $G(\cdot)$ be a right quasigroup in which (1) is true. Then $G(\cdot)$ is an iso-lip loop.

Proof. By Theorem $1, G(\cdot)$ is an iso-lip groupoid. Consequently $a * x=b$ is solvable uniquely for $x$ in $G(*)$, the associated lip groupoid with unity. As $G(\cdot)$ is a right quasigroup, $y \cdot a=b$ is uniquely solvable for $y$ in $G(\cdot)$. This leads to the solvability of $y * a=b$ for $y$ in $G(\cdot)$ showing, thereby, that $G(*)$ is a lip loop. Thus $G(\cdot)$ is an iso-lip loop.

Now, we merely state the corresponding results for rip.

THEOREM 2. A groupoid $G(\cdot)$ is an iso-rip groupoid if, and only if,

$$
(w w) \cdot[\{(y x \cdot u u) \cdot(x \cdot t t)\} \cdot(v v)]=y
$$

or

$$
[(w w) \cdot\{(y x \cdot u u) \cdot(x \cdot t t)\}] \cdot(v v)=y
$$

is true for all $x, y, t, u, v, w \in G$.

Corollary 2. Let $G(\cdot)$ be a left quasigroup in which (12) is true. Then $G(\cdot)$ is an iso-rip loop.

3. Let $G(\cdot)$ be a groupoid. Then $G(\cdot)$ is called an iso-CI loop, if there is a CI loop $G(*)$ which is a principal isotope of $G(\cdot)$ such that $\cdot$ and 
* are connected either by $x * y=\rho(x) \cdot y$ or by $x * y=x \cdot \lambda(y)$ where $\lambda$ is the left inverse operator of $G(*)$ and $\rho=\lambda^{-1}$, is the right inverse mapping.

THeOREM 3. Let $G(\cdot)$ be a right quasigroup. Then $G(\cdot)$ is an iso-CI loop, provided

$$
x \cdot(y x)=y \cdot(t t),
$$

holds for all $x, y, t \in G$ and conversely if $G(\cdot)$ is an iso-CI loop, then (13) holds for all $x, y, t \in G$.

Proof. Let $a \cdot r=a \cdot s$. Then, first with $y=a, x=r$ in (13) and then with $y=a, x=s$ in (13), we get $r \cdot(a r)=a \cdot(t t)=s \cdot(a s)=s \cdot(a r)$. Since $G(\cdot)$ is a right quasigroup, we obtain $r=s$, that is, the left cancellativity of $(\cdot)$.

Then from (13), by keeping $x$ and $y$ fixed and using the left cancellativity we have

$$
t \cdot t=\text { constant }=e(\text { say }), \text { for every } t \in G .
$$

So, (13) becomes

$$
x \cdot(y x)=y e, \text { for all } x, y \in G .
$$

Since $G(\cdot)$ is a right quasigroup, from (15) we see that $a x=b$ implies $a e=x b$ and is then uniquely solvable for $x$. Thus $G(\cdot)$ is a quasigroup. With $y=e,(15)$ gives, using (14), that $x \cdot(e x)=e$, that is,

$$
e x=x \text {, for all } x \in G \text {. }
$$

Now, consider the mapping $\lambda: G \rightarrow G$, such that

$$
\lambda(x)=x e, \text { for all } x \in G .
$$

Then $\lambda$ is a permutation of $G$.

Now, define $G(*)$ as follows:

$$
x * y=\rho(x) \cdot y, \text { for all } x, y \in G,
$$

where $\rho=\lambda^{-1}$. Then $G(*)$ is a principal isotope of $G(\cdot)$ and $G(*)$ is a quasigroup.

$$
\begin{aligned}
& x=e \text { in (18) with (16) gives } e * y=e \cdot y=y, \text { for all } y \in G . \\
& y=e \text { in (18) with (17) gives } x * e=\rho(x) \cdot e=x, \text { for all } x \in G .
\end{aligned}
$$

Therefore, $e$ is the identity of $G(*)$ and hence $G(*)$ is a loop.

From (18), we have

$$
x \cdot y=\lambda(x) * y, \text { for } x, y \in G .
$$

Making use of (17) and (19), (15) becomes, $x \cdot(y x)=\lambda(y)$, that is, 
$x \cdot[\lambda(y) * x]=\lambda(y)$, that is,

$$
\lambda(x) *(y * x)=y, \text { for all } x, y \in G .
$$

Hence $G(*)$ is a CI loop.

Conversely, let the right quasigroup $G(\cdot)$ be an iso-CI loop. Let $G(*)$ be the corresponding CI loop such that $(*)$ and $(\cdot)$ are connected by (18) or (19), where $\lambda$ and $\rho$ are the left and right inverse operators of $G(*)$.

Then (20) holds good in $G(*)$. Now, $y=e$ in (20) gives $\lambda(x) * x=e$. Thus from (19) with $y=x$, we obtain (14).

Also, $y=e$ in (19) gives (17). It is now easy to see from (20), that (13) is true.

Thus the proof of this theorem is complete.

TheOREM 4. Let $G(\cdot)$ be a left quasigroup. Then $G(\cdot)$ is an iso-CI loop, if and only if

$$
x y \cdot x=(t t) \cdot x
$$

is satisfied for all $x, y, t \in G$.

The proof of Theorem 4 is similar to that of Theorem 3.

REMARK. Whereas the isotopy $x * y=\rho(x) \cdot y$ is taken into account in Theorem 3, the isotopy $x * y=x \cdot \lambda(y)$ is to be used in Theorem 4 .

A loop $G(\cdot)$ with identity $e$ is said to be of exponent 2 if $x \cdot x=e$ for all $x \in G$. if

THEOREM 5. A groupoid $G(\cdot)$ is a CI loop of exponent 2 if and only

$$
x \cdot(y x \cdot u u)=y, \text { for all } x, y, u \in G .
$$

Proof. We will show that under the assumption of $(22),(\cdot)$ is both right and left cancellative.

Let $v a=w a$. Then in (22), with $x=a, y=v$ first and then $x=a, y=w$, we get $v=w$. So, $(\cdot)$ is right cancellative. Let $a v=a w$. Setting $x=w$, $y=a$ and $x=v, y=a$ in (22), we obtain $w \cdot[a w \cdot u u]=a=v \cdot[a v \cdot u u]$, from which and the right cancellativity of $(\cdot)$, we get the left cancellativity of $(\cdot)$.

Changing only $u$ in (22) and using the cancellativities of $(\cdot)$, we have

$$
u u=\text { constant }=e(\text { say }), \text { for all } u \in G .
$$

Now, (22) becomes

$$
x \cdot(y x \cdot e)=y, \text { for all } x, y \in G .
$$


Put $y=x$ in (24) to get, with the use of (23), that

$$
x e=x \text {, for all } x \in G,
$$

so that (24) takes the form,

$$
x \cdot y x=y, \text { for all } x, y \in G .
$$

From (25) and (26) with $x=e$, we get

$$
e y=y \text {, for all } y \in G,
$$

showing thereby that $e$ is the identity of $G(\cdot)$. By (23), it follows that $G(\cdot)$ is of exponent 2 and by $(26)$ it follows that $G(\cdot)$ is a CI loop. The converse is trivial. This completes the proof of this theorem.

I express my sincere thanks to the referee for the useful suggestions which improved the presentation of the paper.

\section{REFERENCES}

1. R. Artzy, Crossed-inverse and related loops, Trans. Amer. Math. Soc. 91 (1959), 480-492. MR 21 \#5688.

2. R. H. Bruck, A survey of binary systems, Springer-Verlag, Berlin and New York, 1966.

3. G. Higmann and B. H. Neumann, Groups as groupoids with one law, Publ. Math. Debrecen 2 (1952), 215-221. MR 15, 284.

4. PI. Kannappan, On weak inverse property loops, J. London Math. Soc. 5 (1972), 298-302.

5. ——, Groupoids and groups, Jber. Deutsch. Math. Verein. (to appear).

6. R. Padmanabhan, Inverse loops as groupoids with one law, J. London Math. Soc. (2) 1 (1969), 203-206. MR 39 \#5744.

Faculty of Mathematics, University of Waterloo, Waterloo, Ontario, CANADA 